\title{
NOTAS
}

\section{Erwin Kempton Mapes $(1884-1961)$}

E L I8 de febrero del corriente año dejó de existir en Iowa City el doctor Erwin Kempton Mapes, distinguido profesor de la State University of Iowa y uno de los investigadores norteamericanos más destacados en el campo de la literatura hispanoamericana.

Hijo de Perry Mapes y de Diana Luse, E. K. Mapes nació en un arraigado hogar del Medio-Oeste norteamericano, en Gilman, Illinois, el 9 de junio de r884. También en el Medio-Oeste hizo sus estudios primarios y secundarios. Siendo aún niño fue trasladado al Estado de Iowa, donde vivió casi todo el resto de su vida. Completó sus estudios secundarios en la Tobin Academy de Fort Dodge, Iowa, y obtuvo el grado de bachiller en el Cornell College, de Mount Vernon, también de Iowa, en I909. Pasó luego a la Universidad de Harvard, donde obtuvo el título de "master" (equivalente al de licenciado o profesor de Estado), en I915. En Harvard tuvo oportunidad de conocer al famoso hispanista norteamericano $\mathrm{J}$. D. M. Ford, de quien recibió sus primeras orientaciones hacia los estudios hispanoamericanos.

Con el título otorgado por la Universidad de Harvard pudo iniciarse en la carrera docente. De I9I5 a I9I7 enseñó en el Western State College, de Gunnison, Colorado, y de I9I7 a 1920, en el Westminster College, de Fulton, Misuri. En r9i 8 hizo un viaje, con fines educacionales, a Cuba. Resultado de dicho viaje fue su libro Cuba y los cubanos, publicado en Nueva York en 1920. Este año viajó a Francia, donde, después de un breve paso por la Universidad de Grenoble, en I920, se trasladó a París, en cuya Universidad asiștió a los cursos lectivos de 1920-192 I y de 
I924-I925. En los veranos de I92r, I924 y 1925, el profesor Mapes viajó a Madrid, donde frecuentó el prestigioso Centro de Estudios Históricos y conoció a las personalidades sobresalientes del mismo, como don Ramón Menéndez Pidal, Américo Castro y Tomás Navarro Tomás, entre otras. En la Biblioteca Nacional de Madrid tuvo tratos con su director, el erudito Federico Morcuende, y con Manuel Machado, uno de los más caracterizados poetas del modernismo español, quien fue bibliotecario de la mencionada biblioteca primero y luego de la Biblioteca y Museo Municipales, también de Madrid. Estos viajes de estudio e investigación tuvieton que ser temporarios debido a que el profesor Mapes enseñó de I92 I a I925 en el Cornell College de Iowa. En r925 sostuvo en la Universidad de París su tesis sobre L'influence française dans l'oeuvre de Rubén Dario, con la que obtuvo su título de doctor en letras. Ese mismo año dicha tesis fue publicada por la Librairie Ancienne Honoré Champion, en la importante colección de la Bibliothèque de la Revue de Littérature Comparée dirigida por Fernand Baldensperger y Paul Hazard.

De regreso a su país, también en I925, el doctor Mapes se incorporó a la Universidad de Iowa como profesor asociado del Departamento de Lenguas Romances. $\mathrm{Y}$ a ésta -desde entonces su Universidad-consagró Mapes su vida de maestro, de investigador y de difusor de la cultura y las letras de la América Hispánica. En 1937 fue ascendido a "fullprofessor", que es el rango máximo que otorgan las universidades norteame-

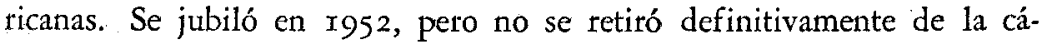
tedra ni de la investigación. El ya venerable profesor "Emeritus" siguió dictando un curso de literatura hispanoamericana, rodeado del respeto y del cariño de sus colegas y alumnos. Asimismo, todos los días concurría a su oficina de Schaeffer Hall, donde el doctor Mapes fue acumulando, durante años de paciente búsqueda, un considerable archivo de materiales microfilmados, en su mayor parte publicaciones dispersas en periódicos de difícil acceso, de escritores modernistas.

En I9I5 E. K. Mapes contrajo matrimonio con Laura Hinkhouse, distinguida dama, también del Medio-Oeste norteamericano, quien le dio dos hijas, Amy Virginia, fallecida, y Frances Elinor, hoy esposa del destacado profesor español de la Brown University, D. Juan López Morillas, quien se doctoró en la Universidad de Iowa con una tesis sobre "El voca, bulario y la dicción de Rubén Darío", bajo la supervisión del doctor Mapes. Tanto la esposa como la hija de Mapes fueron eficaces colaboradoras en tareas inmediatas de su labor de preparación de textos y ediciones. 
La obra dejada por el profesor Mapes comprende dos aspectos igualmente valiosos: el docente y el del investigador.

Como profesor, a partir de su ingreso en la Universidad de Iowa, el doctor Mapes se dedicó con preferencia a la enseñanza de la cultura y de las letras de la América Hispánica, según correspondía a las obligaciones de su puesto. Pero no ha sido uno de esos catedráticos rutinarios y estancados que a menudo hallamos en los ambientes académicos, sino un verdadero educador abierto al progreso y a las nuevas ideas que enriquecen el espíritu y promueven el avance de las instituciones. La State University of Iowa tenía, a la llegada de Mapes, una fuerte orientación española medievalista. En I92I se incorporó a su Departamento de Lenguas Romances el doctor Ralph Emerson House, también oriundo de Iowa, quien fundó y dirigió la conocida serie "University of Iowa Studies in Spanish Language and Literature". La lista de publicaciones de esta colección nos da una idea del tipo de trabajo que alentaba el profesor House, siguiendo rutas señaladas por su maestro de la Universidad de Chicago, el doctor Karl Pietsch. Son, en su mayoría, ediciones críticas de obras españolas antiguas, preparadas previamente como tesis doctorales por alumnos de la sección española. La intervención del doctor Mapes permitió ampliar el campo de los estudios hispánicos con la extensión a la literatura española más moderna (Siglo xix con preferencia) y a la literatura hispanoamericana, con especial énfasis en el modernismo y escritores del siglo xx. Una docena de tesis doctorales dan testimonio de la importante contribución de nuestro colega, director paciente y cuidadoso de la formación de sus alumnos. Destacamos, entre otras, por la indudable seriedad del método y la aportación que ellas significan, las tesis siguientes: Arthur B. Conner, Indications in the Writings of Manuel Gutierrez Nájera of His Reading in French Literature; Juan López-Morillas, El vocabulario y la dicción de Rubén Dario; George Oswald Schanzer, Vida $y$ obra de Ennesto Herrera, y George S. Ulibarri, The Gamonal in the Spanish American Novel of Social Protest. Y como el profesor Mapes hablaba, leía y escribía el francés a la perfección, también dirigió tesis en la sección francesa, algunas de las cuales fueron indispensable labor preparatoria para el conocimiento de las influencias que incidieron en la literatura hispanoamericana. Destaco la disertación del doctor Camile Le Vois, actual profesor de la Universidad de Iowa, titulada La Cbronique parisienne pendant la dernière partie du dix-neuvième siècle.

En el orden de la investigación, Mapes se destaca como uno de los más nobles y esforzados pioneros norteamericanos que al promediar el 
primer cuarto del siglo abrieron rutas firmes para el conocimiento de Nuestra América. Pertenece, por tanto, a la primera generación de hispanoamericanistas de Estados Unidos, país que marcha a la vanguardia en estos estudios. Mapes forma, con Isaac Goldberg, Alfred Coester, Aurelio M. Espinosa, padre, y el dominicano Pedro Henríquez Ureña, entre los de mayor prestigio internacional, el grupo de los fundadores del hispanoamericanismo literario norteamericano, abuelos ilustres que comparten con historiadores antropólogos, etnólogos, etc. la magna tarea de descubrirnos y valorarnos en lo que somos. ${ }^{1}$ De ellos vienen los de la promoción que llamaremos los padres, con denominación que agradaba al profesor Mapes: Waldo Frank, Holmes, Leonard, Leavitt, Spell, Reid, Van Horne, Umphrey, Hespelt, Torres-Rioseco, García-Prada, Englekirk, Manuel Pedro González y muchos más, en cuya acción cultural se advierte ya la intima colaboración entre norteamericanos e hispanoamericanos. $Y$ dejamos para un trabajo especial la lista completa, incluyendo la tercera generación, la de los hijos, y acaso la cuarta, la de los nietos, porque el recuento sería interminable. Todos estos generosos comprendedores de la llamada América Latina han convertido a Estados Unidos, repito, en el primer país hispanoamericanista de este siglo. Cada uno de ellos, en su campo de especialización, ha hecho resonar con gloria y honor en todo el mundo el departamento de español o de lenguas romances de la universidad a que pertenecieron. La Universidad de Iowa tiene gran parte de esta deuda contraída con el doctor Mapes, cuyo nombre llega a mi recuerdo desde mis años de estudiante en la Universidad de Buenos Aires.

La reputación internacional del doctor Mapes comienza con su tesis sobre L'influence française dans l'oeuvre de Rubén Dario (París, 1925). Mapes estudió en la Universidad de París con los profesores Baldensperger, Hazard y Martinenche, especialistas en literaturas comparadas, sobre todo Baldensperger, maestro de Hazard. La influencia del comparatismo es evidente en aquel primer libro de Mapes, tanto en el método como en la concepción del hecho literario. El método es descriptivo, derivado del positivismo. El tratamiento del hecho literario depende de la creencia de que la creación poética es una síntesis final del proceso cultural. De ahí el énfasis puesto en el estudio de las influencias. Mapes define su posición cuando dice: ". . un des aspects les plus importants de l'art de Darío [es] son adaptation à l'espagnol des procédés techniques de certains auteurs français de son siècle. C'est donc à des recherches sur cette partie de

1. Preciso es recordar que detrás de estos iniciadores estaba el gran hispanista J. D. M. Ford, promotor de evocaciones y fundador de "The Harvard Council on Hispano-American Studies". 
l'oeuvre du poète, que je veux consacrer le travail que voici" (op. cit., "Avant-Propos", p. V). Acerca del modernismo dice: "Le fond même du Modernisme consistait à adapter à l'espagnol, et à fondre en un tout assez harmonieux, un grand nombre de procédés employés par plusieurs école françaises du XIXe siècle, notamment par le Romanticisme, le Parnasse et le Symbolisme" ( $I d$, chap. I, P. I).

Los resultados obtenidos por este medio, en especial la comprobación de los antecedentes franceses de técnicas y procedimientos de Rubén Darío, fueron cuestionados por investigadores hispanoamericanos, sobre todo por Arturo Torres-Ríoseco, quien escribió otra tesis doctoral para mostrar que muchas de las llamadas por Mapes "influencias francesas" ya se encontraban en la antigua tradición española. Otros críticos, como Pedro Henríquez Ureña, Raúl Silva-Castro, Arturo Marasso, Julio Saavedra Molina, Diego Manuel Sequeira, Ernesto Mejía Sánchez, etc., han corregido y completado muchos aspectos de la labor de Mapes. Pero el libro de Mapes debe ser considerado como la primera obra metódica que promovió las más serias investigaciones sobre el gran poeta nicaragüense. El mismo Mapes ha contribuido con nuevos estudios al esclarecimiento de la verdadera originalidad de Darío.

La obra posterior del profesor Mapes se ha ceñido al método histórico más objetivo, en el que muy pocas veces se hace necesaria la intervención de la sensibilidad y la valoración estética consecuente. Es una labor de "scholar" puro, de erudito imparcial y enemigo de cualquier riesgo crítico sólo asentado en la inferencia o el descubrimiento personal y creativo de la crítica artística y de la estructuración de amplios cuadros ideológicos. La experiencia, madurada en nuevos y fecundos aprendizajes, siempre renovados, convirtió a este probo maestzo en un espíritu circunspecto y en un dador humilde, con sincera modestia, de materiales de primera mano y únicamente seguros. Mapes ha dedicado sus años maduros a una infatigable labor de pesquisa, dirigida a descubrir textos, a aclarar seudónimos y a fijar fechas, antecedentes y otros aspectos oscuros de escritores modernistas. $\mathrm{Y}$ ha dejado una respetable labor de recopilación de escritos inéditos o de páginas olvidadas en diarios y revistas de difícil acceso; Mapes compara versiones, infiere críticamente, demuestra con hechos, depura, anota, edita. Estamos ahora en presencia del especialista riguroso, que sigue las huellas de un autor en la búsqueda retrospectiva, con esa perseverancia y minuciosidad que agota las fuentes documentales y acarrea el máximo de evidencias demostrativas. En este sentido su aportación al conocimiento de obras inéditas, desconocidas u olvidadas de Rubén Darío y Manuel Gutiérrez Nájera es fundamental, así como la 
precisión de muchos seudónimos de Gutiérrez Nájera y la preparación de casi todos los textos para una edición de las Obras del poeta mexicano, labor realizada en Iowa y entregada para su edición definitiva a la Universidad de México.

La obra de investigación del doctor Mapes ha contado con el auspicio y subsidio de varias instituciones norteamericanas: en 1936 lo becó el American Council of Learned Societies; en I938, la American Philosophical Society, y posteriormente, en dos oportunidades, fue ayudado económicamente por la State University of Iowa. Así pudo Mapes viajar a Argentina y México y aplicar su personal método de fotocopiar periódicos y revistas.

Mapes ha sido miembro del Instituto Internacional de Literatura Iberoamericana, vice-presidente de su Mesa Directiva durante el período de 1939-1940, y presidente del Comité Permanente dé Coordinación de Investigaciones de dicho Instituto desde 1940. A propuesta de Alfonso Reyes, la Academia Mexicana de la Lengua le otorgó "Diploma de Honor" en r940. El Departamento de Estado de los Estados Unidos lo llamó a colaborar en la preparación de textos para la enseñanza del inglés en Hispanoamérica desde 1940 a 1946. Pertenecía a la American Association of University Professors, a la Asociación Americana de Profesores de Español y Portugués, a la Sociedad de Profesores de Lenguas Modernas de los EE. UU., y a la Sociedad Norteamericana de la Historia. La John Hay. Whitney Foundation lo nombró profesor visitante durante el año lectivo de $1952-1953$.

Mapes ha colaborado en las siguientes prestigiosas revistas: Hispanic Review, Revista Hispánica Moderna, Hispania, Revista Iberoamericana, Philological Quaterly, PMLA, Books Abroad, Modern Language Journal, Symposium, Revue de Littérature Comparée y Revista Chilena de Historia y Geografía.

De Mapes podemos decir, sin exageración: fue una vida consagrada a los bienes de la cultura. El contacto con grandes maestros en los mejores centros de trabajo y estudio de su especialidad, ampliaron el campo de su visión cultural y le aseguraron un método de investigación y una orientación altamente científica. Apasionado de las cosas de la América española hizo por ella cuanto puede hacer un esforzado paladín de los ideales que abraza y la vocación que lo anima. Callado, minucioso, sereno, fue siempre amable y generoso con quienes se le acercaban en busca de su ayuda y consejo. Amó a sus alumnos y apoyó siempre a los jóvenes. Nunca se asustó cuando éstos venían, impetuosos e inexpertos, a querer barrer con las murallas del conservadorismo local. Espíritu cosmopolita, supo 
desmentir constantemente el tan mentado provincialismo del Medio-Oeste norteamericano. Acogió lo nuevo y se renovó. Fue, por eso, más digno por lo que comprendió y admitió que por lo mucho que él logró dar de sí. Esta es su gran lección, su ejemplo que ojalá pudiéramos seguir.

\section{OBRA PUBLICADA Y I.ABOR PEDAGOGICA DEL DOCTOR MAPES}

\section{A. LIBROS Y ARTículos:}

Cuba y los cubanos (New York: Gregg Publishing Co., 1920).

L'influence française dans l'oeuvre de Rubén Dario (París: Librairie Ancienne Honoré Champion, 1925).

"A survey course and period courses in Spanish-American literature", Modern Language Journal, noviembre 1929.

"The teaching of Spanish-American literature in the United States", Hispania, noviembre 193 r.

The essentials of Spanish grammar [con R. E. House] (Ginn and Co., 1932).

"Innovation and French influence in the metrics of Rubén Dario", PMLA, marzo I934 [En español: "Innovación e influencia francesa en la métrica de Rubén Darío", Revista Hispánica Moderna, enero 1940].

"Rubén Dario's first sonnets in alexandrines", Pbilological Quaterly, enero I935 [En español: "Los primeros sonetos alejandrinos de Rubén Darío", Revista Hispánica Moderna, julio I935].

"El modernismo en las letras chilenas", Revista Cbilena de Historia y Geografia, Santiago, Chile, abril I935.

"Recent research on the modernist poets", Hispanic Review, enero 1936.

"The first published writings of Manuel Gutiérrez Nájera", Hispanic Review, julio 1937. [En español: "Primeros escritos publicados de Manuel Gutiérrez Nájera", Universidad, México, enero-febrero 1937].

"Implications of some recent studies on style", Revue de Littérature Comparée, París, julio-septiembre 1938.

Escritos inéditos de Rubén Dario (New York, Columbia University, Hispanic Institute, 1938).

'Una edición conmemorativa de las obras de Rubén Darío publicadas en 
Chile", Memoria del primer congreso internacional de literatura iberoamericana, México, I938.

Obras inéditas de Manuel Gutiérrez Nájera: Crónicas de "Puck" (New York, Columbia University, Hispanic Institute, 1939).

Obras escogidas de Rubén Dario publicadas en Chile [con Julio Saavedra Molina] (Santiago, Chile: imprenta de la Universidad de Chile, 1939.)

Sborter Spanish Grammar [con R. E. y R. D. House] (Ginn and Co., I94 $\mathrm{r}$ ).

"Foreword" [nota necrológica sobre R. H. House], Pbilological Quaterly, vol. XXI, No. I, enero I942.

$Y$ va de cuento [con Juan López Morillas] (Ginn and Co., I943).

Obras inéditas de Manuel Gutiérrez Nájera: Poesías (New York: Columbia University, Hispanic Institute, I943).

"Teaching modern languages in war time", Madern Language Joumal, diciembre 1943 .

"Bibliografía de tesis sobre literatura iberoamericana preparadas en las universidades de Iberoamérica", Revista lberoamericand, vol. VI, No. II, febreto I943, pp. 203-206.

History of the National Library of México [traducción inglesa de Historia de la Biblioteca Nacional de México, por Rafael Carrasco Puente] (México, r948).

"The pseudonyms of Manuel Gutiérrez Nájera",PMLA, septiembre I949. [En español: "Los seudónimos de Manuel Gutiérrez Nájera", Revista Hispánica Moderna, enero-diciembre I953; completado con: "Bibliografía periodística de Manuel Gutiérrez Nájera" (Id.) ]

$Y$ va de cuento. Alternate [con Manuel López Morillas] (Ginn and Co., I950).

El Periquillo Samiento [con Frances M. López Morillas]; edición abrevia$\mathrm{da}$, con notas y vocabulario, para uso escolar (Appleton-CenturyCrofts, I952).

El tesoro de Cuaubtémoc [con R. Davis]; edición abreviada, con notas y vocabulario, para uso escolar (Ginn and Co., r953).

Brief course in Spanish [con Ruth House Weber] (Boston: Ginn, I955). Manuel Gutiérrez Nájera, Cuentos completos y otras narraciones, Prólogo, edición y notas de E. K. Mapes. Estudio preliminar de Francisco González Guerrero (México: Fondo de Cultura Económica [Biblioteca Americana, 35], 1958 .

Manuel Gutiérrez Nájera, Obras. Crítica literaria, I. Ideas y temas literarios. 
Literatura mexicana. Investigación y recopilación de E. K. Mapes. Edición y notas de Ernesto Mejía Sánchez. Introducción de Porfirio Martínez Peñaloza. (México: Universidad Nacional Autónoma de México. [Centro de Estudios Literarios. Nueva Biblioteca Mexicana, 4], I959).

B. Reseñas (lista parcial).

"Selections from prose and poetry of Rubén Dario" [de Umphrey y García-Prada], en Hispania, mayo r929.

"Parnassus in France" [de Aaron Schaffer], en Pbilological Quaterly, octubre $193 \mathrm{r}$.

"Obras desconocidas de Rubén Dario" [de Raúl Silva-Castro], en Books Abroad, Verano 1935 y en Revista Hispánica Moderna, abril 1935.

"Rubén Darío y la creación poética" [de Arturo Marasso], en Books Abroad, Verano 1935 y en Revista Hispánica Moderna, abril 1935.

"Artículos históricos-literarios" [de Narciso Alonso Cortés], en Hispania, mayo 1935 .

"The poetry of José Mármol" [de Stuart Cuthbertson], en Hispanic Review, abril 1936.

"A bibliography of Rubén Dario" [de Henry Grattan Doyle], en Hispanic Review, julio 1936.

"Amado Nervo: México's religious poet" [de E. Turner Wellman], en Hispanic Review, octubre 1937.

"Bascuñán, el cautivo" [de Alejandro Vicuña], Books Abroad, octubre I949.

"Cuentos completos de Rubén Dario" [ed. de Ernesto Mejía Sánchez y prólogo de Raimundo Lida], Symposium (Syracuse University), I.95I.

"En torno a Gutiérrez Nájera" [de Boyd G. Carter], Revista Iberoamericana, vol. XXV, Núm. 50 (r960), pp. 35r-354.

\section{Tesis Doctorales Dirigidas por el Dr. Mapes}

Conner, Arthur B., Indications in the writings of Manuel Gutierrez of bis readings in French literature (195r).

Dreps, Joseph Antone, The metrics of José de Espronceda (193I). 
Holm, Lydia, The epithet in the works of Ruben Dario (1947).

LeVois, Camille Joseph, The chronique parisienne pendant la derniêre partie du dix-neuvième siècle (I932).

López-Morillas, Juan, El vocabulario y la dicción de Rubén Dario (1940).

McNeill, Mary L., Costumbrismo and folklore in the social novel of the Central Andean Region (1936).

Roberts, Graves Baster, The epithet in Spanish poetry of the romantic period (r934).

Schanzer, George Oswald, Vida y obra de Erniesto Herrera (1950).

Sisto, David Theodore, Character analysis in the works o Carlos Reyles (1935).

Sittler, Richard Culver, The Alexandrine verse form in post-modernist Spat nish American poetry (1952).

Spicer, Julia Racine, The epithet in the parnassian school of French poetry I935).

Ulibarri, George S., The gamonal in the Spanish American novel of social protest (1952).

ALFREDO A. ROGgIANO

State University of lowa 